\title{
Real-Time Interactive Performance QoS Research of the Online Web-game Control System
}

\author{
Qiang Zhang ${ }^{\mathrm{a}}$, Yiquan Kong ${ }^{\mathrm{b}}$ \\ Lingnan Normal University, Guangdong Zhanjiang 524048 China \\ a zjzs509@163.com, b20693458@qq.com
}

Keywords: Real-Time, Web-game, QoS.

\begin{abstract}
The provision of end-to-end Quality of Service (QoS) for real-time interactive online web-game control system over IP-based networks is already an open issue. To achieve this goal, the paper used one method which contained optimization algorithm of real-time interactive online web-game in the model network. Empirical results show that solution guarantees the QoS of the real-time interactive online web-game data stream and enhances the efficiency of network resources real-time interactive communication.
\end{abstract}

\section{Introduction}

An efficient allocation of real-time interactive online web-game control system satisfying QoS requirements is the primary goal of QoS-based multicast routing. However, individual QoS parameters may be conflicting and interdependent, thus making the problem even more challenging [1].

With increasing demand for real-time services in interactive online web-game control system, quality-of-service (QoS)-based routing offers significant challenges. Multimedia applications like executive telepresence systems, real-time streaming of stock quotes or processing of web-game images relayed from satellites require strict QoS guarantee (e.g. bandwidth, delay)while communicating among multiple hosts[2]. Our solution attempts to optimize multiple QoS parameters (e.g. end-to-end delay, bandwidth guarantee and residual bandwidth utilization [3]) simultaneously.

In the paper, we use QoS optimization algorithm, Webpage processing algorithm and other comprehensive technique to develop an efficient solution which determines multicast routes on demand by simultaneously optimizing end-to-end delay guarantee, bandwidth requirements and residual bandwidth utilization without combining them into a single scalar objective function. Using suitable genetic operators, the solution is capable of finding near-optimal solutions within a few iterations. We have shown that with the increase in the number of nodes our solution performs having good optimization. From the experimental results it is clear that our algorithm is capable of obtaining the global optimal values for QoS parameters.

\section{Description of the proposed algorithm}

The Optimization Algorithm of real-time interactive online web-game control system.The QoS optimization algorithm of Voice communication in the telepresence system is based on theoretical evidence that, under assumptions, maximum log-likelihood learning of a mixture can be done in a greedy manner by successively adding components to the mixture and the parameters of optimization algorithm of reverberation data can be estimated.

In particular, assume that a new optimization algorithm component $\varphi\left(\boldsymbol{x}_{n}, \boldsymbol{\beta}_{k+1}\right)$ is added to a presented k-component mixture $f_{k}\left(x_{n}\right)$, then giving the $\mathrm{k}+1$ component mixture $\boldsymbol{f}_{k+1}\left(x_{n}\right)$ like Eq.1.

$$
f_{k+1}\left(x_{n}\right)=(1-\alpha) f_{k}\left(x_{n}\right)+\alpha \varphi\left(x_{n}, \beta_{k+1}\right)
$$

Where $\alpha$ is the weight coefficient whose range between 0 and $1, \boldsymbol{\beta}=\{\boldsymbol{\mu}, \boldsymbol{\alpha}\}$ is optimization algorithm component parameter vector. Then as long as for each $\mathrm{k}$, given $f_{k}\left(x_{n}\right)$. The weight $\alpha$ and the parameter 
vector $\boldsymbol{\beta}_{\boldsymbol{k}+1}$ are optimally chosen so that the new likelihood described as follow is maximized like Eq.2.

$$
L_{k+1}(\theta)=\sum_{n=1}^{N} \ln f_{k+1}\left(x_{n}\right)=\sum_{n=1}^{N} \ln (1-\alpha) f_{k}\left(x_{n}\right)+\alpha \varphi\left(x_{n}, \beta_{k+1}\right)
$$

It can be seen that, unlike other optimization algorithm which starts with a random configuration of all components, the optimization algorithm start with the optimal one-component mixture, whose parameters are trivially computed.

The Webpage processing Algorithm of real-time interactive online web-game control system.The Webpage processing algorithm states that if a page has important links to it, its links to other pages also become important. Therefore, Webpage processing takes the backlinks into account and propagates the ranking through links: a page has a high rank if the sum of the ranks of its backlinks is high.

A slightly simplexes version of Webpage processing is defined as

$$
P R(u)=c \sum_{v \in B(u)} \frac{P R(v)}{N_{V}}
$$

The $u$ represents a web page. $\mathrm{B}(\mathrm{u})$ is the set of pages that point to u.PR $(\mathrm{u})$ and PR $(\mathrm{v})$ are rank scores of page $u$ and $v$, respectively. Nv denotes the number of outgoing links of page $\mathrm{v}$. $\mathrm{c}$ is a factor used for normalization. Figure 4 shows an example in which c $=1.0$ to simplify the calculation.

Based on the consideration of the phenomenon mentioned above, the original Webpage processing is published.

$$
P R(u)=(1-d)+d \sum_{v \in B(u)} \frac{P R(v)}{N_{v}}
$$

Where $d$ is a dampening factor that is usually set to 0.85 . We also could think of $d$ as the probability of users' following the links and could regard $(1-d)$ as the Webpage processing distribution from non-directly linked pages

\section{The network structure of real-time interactive online web-game control system}

Multiprotocol Label Switching (MPLS) can speed up the flow of network traffic and make it easier to manage [4]. MPLS is flexible, fast, and cost-efficient and allows for network segmentation and quality of service (QoS). MPLS also offers a better way of transporting latency-sensitive applications like voice and video. While MPLS technology has been around for several years, businesses are now taking advantage of service provider offerings and beginning their own corporate implementations. Our model network used in this proposal is shown in Fig. 1.

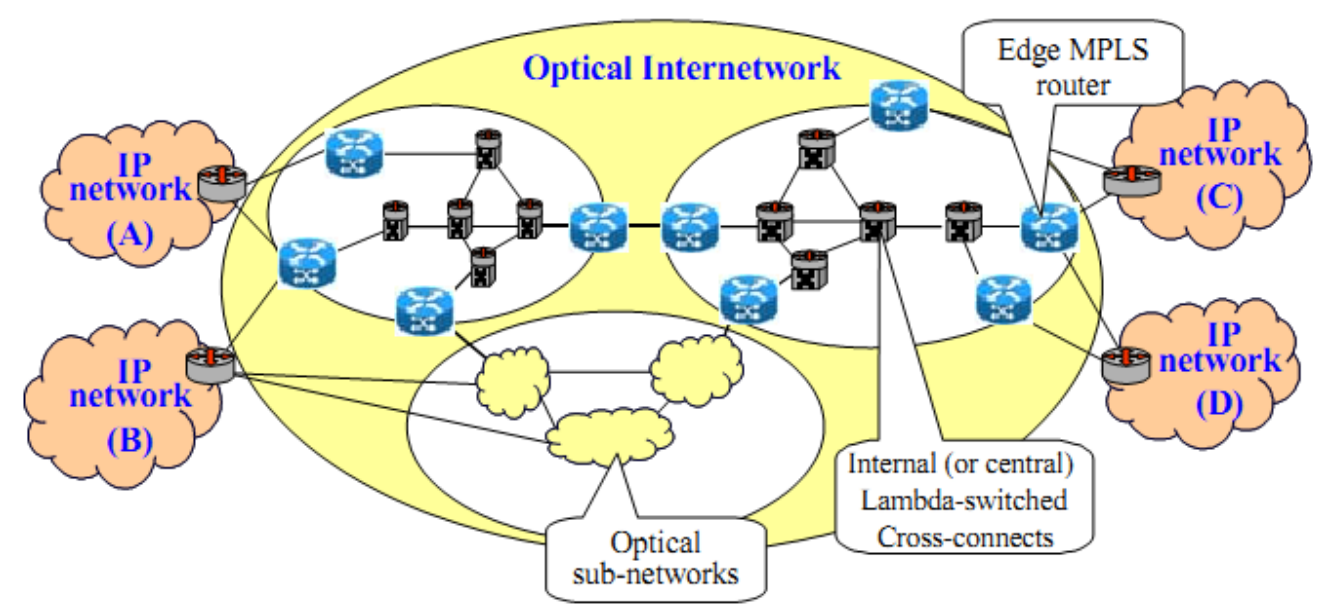


Fig. 1. Model network used in this proposal

MPLS is a versatile solution to address the problems faced by present-day networks-speed, calability, quality-of-service (QoS) management, and traffic engineering. MPLS has emerged as an elegant solution to meet the bandwidth-management and service requirements for next-generation IP-based backbone networks.

Multi-Protocol Label Switching (MPLS) TE provides a more elegant and efficient technique than IP source routing. It allows traffic travel down a path different from conventional Interior Gateway Protocol (IGP) destination based hop-by-hop routing [5]. The path is pre-determined at tunnel setup time. Routers along the path do not have to examine the IP header of every passing packet. The basic idea of MPLS involves assigning short fixed length labels to packets inside an MPLS cloud. Throughout the MPLS domains, the labels attached to packets are used to make forwarding decisions. It allows decoupling of the information used for forwarding (a label) from the information carried in the IP header. MPLS TE, using the RSVP signaling mechanism, injects the notion of a connection to connectionless IP through nailed-up label switched paths (LSP). MPLS TE provides capabilities to specify an explicit path for the LSP before it is established. We will refer the nailed-up LSP as an MPLS TE tunnel, or simply tunnel.

\section{Experiment and Analysis}

In the real-time interactive online web-game control system experiment, we use MOGA algorithms. The blessing of multi-objective-genetic algorithms (MOGA) has paved the way to develop the algorithm for a new QoS-based multicast on-demand routing algorithm. The mathematical analysis shows the power of selection and complexity of the algorithm.

The experiment judges the procedure realization application effect through the network test response time. Our experimental topology is shown in Fig. 2.

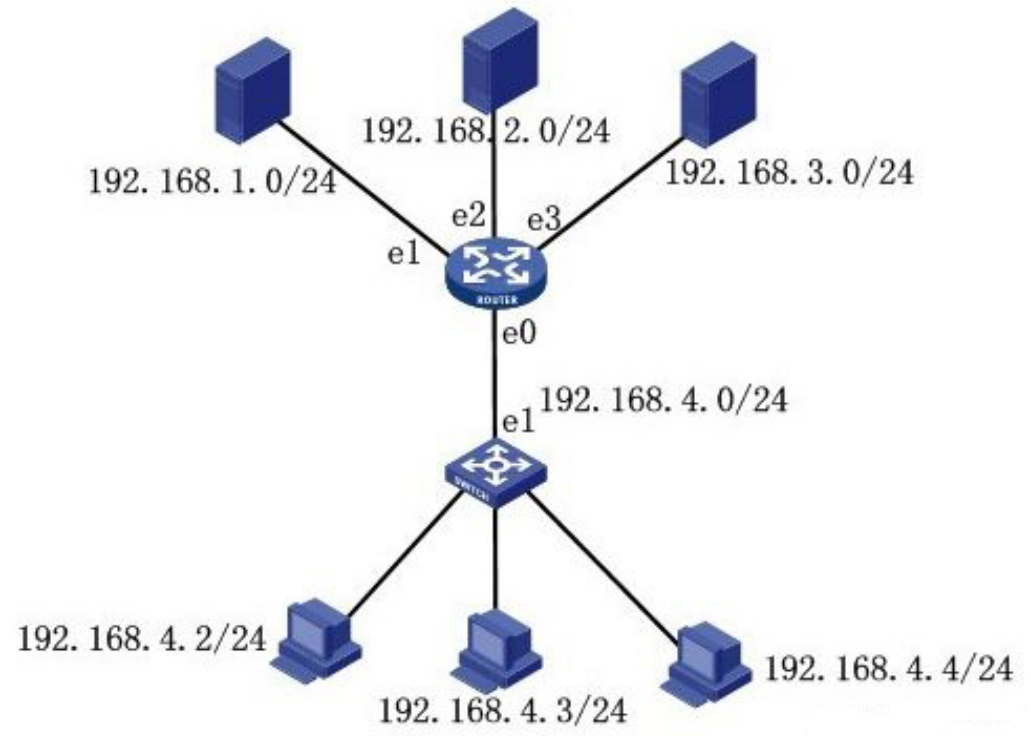

Fig. 2. Model network used in this proposal

The network has eight kinds equipment of network, including router, switch, pc and servers. Each node represents capital terminal equipment. In the simulations, model network links were assumed. Their propagation delays in milliseconds are listed. These delays are based in real distances.

The experimental result in Fig. 3 shows the effect of our solution in the real-time interactive online web-game control system. In the experiment, we simulated five data streams. In the simulated network, we tested the quality of web-game packets by using professional server network software wireshark module accurately in both the upstream and downstream directions. We measure the critical performance metrics such as SIP, Jitter, packet loss, and loss distribution and delivers score result for a wide range of popular web-game codecs in our solution which shows well.

The real-time multimedia data stream flow all through the UDP protocol transmission is able to provide effective equity protection. The solution improved network throughput significantly reduced 
the packet loss and delay of data packets. It enhances the efficiency of network resources and guarantees the QoS of the web-game data stream. The simulated results of real-time interactive online web-game data stream are better than calculated results in our experiment.

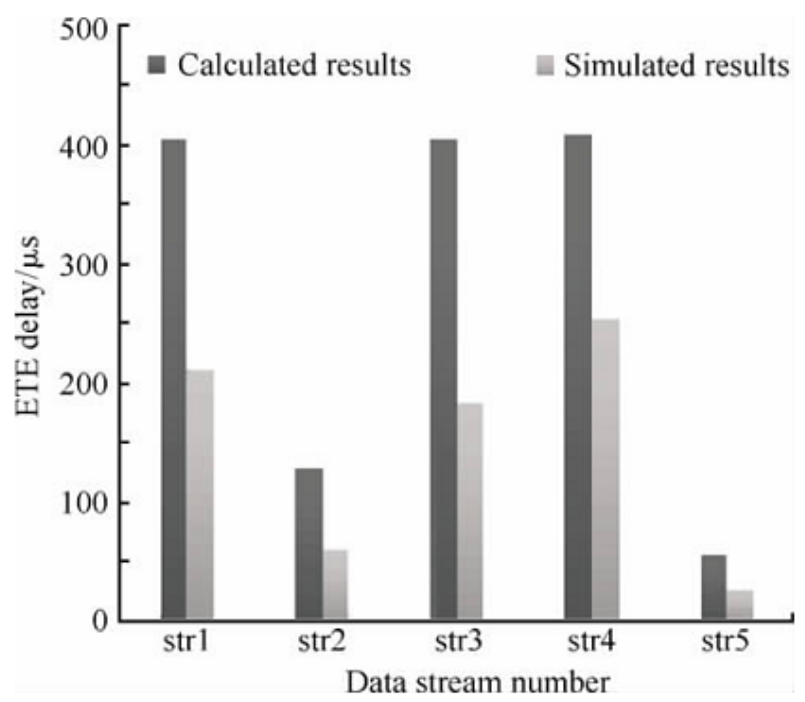

Fig.3 Experimental QoS Result

In the simulated experiment, empirical results show that solution guarantees the QoS of the real-time interactive online web-game data stream and enhances the efficiency of network resources real-time interactive communication. And we achieved the provision of end-to-end Quality of Service for real-time interactive online web-game control system over IP-based networks using our method which contained optimization algorithm of real-time interactive online web-game in the model network.

\section{Summary}

Recent years have witnessed a tremendous growth in the use of real-time interactive online web-game control system around the world.With the growing demand for integrated services supporting multimedia in real-time interactive communication systems, quality of service (QoS) provisioning in real-time interactive communication systems networks is becoming increasingly important. Since bandwidth is one of the most precious resources in the systems, an efficient call admission control scheme is very important to guarantee QoS and to maximize radio resource utilization simultaneously [6]. In end-to-end Quality of Service (QoS) scenarios, these real-time guarantees required by the interactive online web-game systems could be achieved using QoS differentiation between traffic classes over heterogeneous networks. On the other hand, with the aim of reducing the huge amount of information generated by the data stream source, loss compression techniques are applied.

\section{References}

[1] D. Wu, Y.T. Hou, W. Zhu, Y. Zang, and J.M. Peha: IEEE Trans. on Circuits and Systems for Video Technology ,Vol.11 (2001), p. 282..

[2] I.V. Martín, J.J. Alins-Delgado, M. Aguilar-Igartua, and J. Mata-Díaz: 16th IFIP/IEEE Distributed Systems: Operations and Management, LNCS, Vol.3775 (2005), p. 157.

[3] G.M. Muntean and L. Murph: IEEE Transactions on Broadcasting, Vol.50 (2004), p. 1.

[4] Information on http://www.cisco.com

[5] D. H. Lorenz and A. Orda: IEEE/ACM Transactions on Networking, Vol.6 (1998), p. 768.

[6] N. Banerjee and S. K. Das: International Conference for Communication, Vol.8 (2001), p. 2588 